\title{
MATHEMATICAL MODELLING OF PROBLEM ON NON-LINEAR FLUTTER OF VISCO-ELASTIC SHELLS
}

\author{
Bakhtiyar Khudayarov \\ Tashkent institute of irrigation and melioration. \\ UZBEKISTAN, 700135, Tashkent-135, Chilanzar-16-24-56. \\ E-mail: bakht-flpo@yandex.ru
}

\begin{abstract}
In this work is investigated the flutter of viscoelastic cylindrical shell streamlined by gas current. The basic direction of work is consisted in taking into account of viscoelastic material's properties at supersonic speeds. The vibration equations relatively of deflection are described by Integra-differential equations in partial derivatives. By Bubnov-Galerkin methods reduced the problems to investigation of system of ordinary Integro-Differential Equations (IDE). The IDE are solved by numerical method, which based on using of quadrature formula. The algorithm of the numerical solution on the basis of the method was described. Critical speeds for cylindrical shell flutter are defined. The influence of the viscoelastic property of the material, geometrical and aerodynamically non-linearity to the current value of critical speed and amplitude-frequency characteristics of the cylindrical shells was analyzed.
\end{abstract}

Keywords: viscoelastic, flutter, shell, Integro-differential equation

\section{INTRODUCTION}

The problems of algorithmization of mechanics problem of continua are studied in works of the academician of Academy of Sciences of Republic of Uzbekistan V.K.Kabulov [1-3]. In the given article this problem is examined for non-linear problems on flutter of viscous-elastic cylindrical shells.

Earlier in works [4-8] and others flutter of elastic cylindrical panels and shells in a supersonic gas current were examined. For description of elastic behavior of shells the equations of Margerra were used.

In connection with wide introduction of composite materials in the aircraft designs, creation of the calculation methods of separate elements of constructions, made of the materials had great importance [9-11].

In the given work mathematical models of the problems of the visco-elastic shells were constructed by taking account of the geometrical and aerodynamical non-lineariaty, aerodynamical damping on the basis of the Kirchhoff-Love hypothesis.

For the solution to the system was applied the problem was solved by the usual IDE based on flexure polynomial approximation by means of Bubnov-Galerkin's method. The numerical method based on quatrature proposed by F.Badalov and
H.Eshmatov [12, 13]. It was described the algorithm of the numerical solution on the basis of the method.

Critical speeds of the visco-elastic shell flutter moved by constant supersound speed were found in all parts of the problem.

\section{MAIN EQUATIONS}

Let's consider hinget supported, selfcontained, circumferential, visco-elastic cylindrical shell with radius of curvature $R$ of a median surface and length $L$, externally streamlined by a supersonic gas current with speed $V$, directed along generatrix.

The equation of motion of the shell is $[7,8]$

$$
\begin{aligned}
& \frac{D}{h}\left(1-R^{*}\right) \nabla^{4} W=L(W, \Phi)+ \\
& +\frac{1}{R} \frac{\partial^{2} W}{\partial x^{2}}-\rho \frac{\partial^{2} W}{\partial t^{2}}+\frac{q}{h} ; \\
& \frac{1}{E} \nabla^{4} \Phi=-\frac{1}{2}\left(1-R^{*}\right) \times \\
& \times\left\{L(W, W)+\frac{1}{R} \frac{\partial^{2} W}{\partial x}\right\}
\end{aligned}
$$

Where $D=E h^{3} / 12\left(1-\mu^{2}\right)$ is the flexural rigidity, $E$ the Young's modulus, $h$ the shell thickness, and 
$\mu$ the Poisson ratio. In Eq.(1), $\rho$ is the shell material density, $W(x, y, t)-$ is the transverse displacement; $\Phi(x, y, t)$ - Airy stress function .

The biharmonic term is defined as

$$
\nabla^{4}=\frac{\partial^{4}}{\partial x^{4}}+2 \frac{\partial^{4}}{\partial x^{2} \partial y^{2}}+\frac{\partial^{4}}{\partial y^{4}} .
$$

$L-$ the differential operator :

$L(W, \Phi)=\frac{\partial^{2} W}{\partial x^{2}} \frac{\partial^{2} \Phi}{\partial y^{2}}+\frac{\partial^{2} W}{\partial y^{2}} \frac{\partial^{2} \Phi}{\partial x^{2}}-2 \frac{\partial^{2} W}{\partial x \partial y} \frac{\partial^{2} \Phi}{\partial x \partial y}$,

$$
L(W, W)=2\left[\frac{\partial^{2} W}{\partial x^{2}} \frac{\partial^{2} W}{\partial y^{2}}-\left(\frac{\partial^{2} W}{\partial x \partial y}\right)^{2}\right]
$$
from

In Eq.(1), $R^{*}$ is the relaxation operator obtained

$$
R^{*} \psi=\int_{0}^{t} R(t-\tau) \psi(\tau) d \tau
$$

$R(t-\tau)$ the relaxation kernel.

The strain components in each point of shell $\varepsilon_{x}, \varepsilon_{y}, \varepsilon_{x y}$ are connected to stress components $\sigma_{x}, \sigma_{y}$ and $\sigma_{x y}$ relations:

$$
\begin{aligned}
& \varepsilon_{x}=\frac{1}{E}\left(1+\Pi^{*}\right)\left(\sigma_{x}-\mu \sigma_{y}\right) \\
& \varepsilon_{y}=\frac{1}{E}\left(1+\Pi^{*}\right)\left(\sigma_{y}-\mu \sigma_{x}\right) \\
& \varepsilon_{x y}=\frac{2(1+\mu)}{E}\left(1+\Pi^{*}\right) \sigma_{x y}
\end{aligned}
$$
[7]:

The inverse relationships is given by as follows

$$
\begin{aligned}
\sigma_{x} & =\frac{E}{1-\mu^{2}}\left(1-R^{*}\right)\left(\varepsilon_{x}+\mu \varepsilon_{y}\right), \\
\sigma_{y} & =\frac{E}{1-\mu^{2}}=\left(1-R^{*}\right)\left(\varepsilon_{y}+\mu \varepsilon_{x}\right), \\
\sigma_{x y} & =\frac{E}{2(1+\mu)}\left(1-R^{*}\right) \varepsilon_{x y},
\end{aligned}
$$

$$
\frac{1}{E}\left(1+\Pi^{*}\right)=\left[E\left(1-R^{*}\right)\right]^{-1}-\text { the operators are }
$$

mutually return.

Let's express now moments

$$
M_{x}, M_{y} \text { and } M_{x y}
$$

through function of $\mathrm{W}(\mathrm{x}, \mathrm{y}, \mathrm{t})$ is the transverse displacement [8]:

$$
\begin{aligned}
& M_{x}=-D\left(1-R^{*}\right)\left(\frac{\partial^{2} W}{\partial x^{2}}+\mu \frac{\partial^{2} W}{\partial y^{2}}\right), \\
& M_{y}=-D\left(1-R^{*}\right)\left(\frac{\partial^{2} W}{\partial y^{2}}+\mu \frac{\partial^{2} W}{\partial x^{2}}\right), \\
& M_{x y}=D(1-\mu)\left(1-R^{*}\right) \frac{\partial^{2} W}{\partial x \partial y} .
\end{aligned}
$$

The forces per unit length in the $x$ and $y$ axis directions, as well as the shear force, are given by

$$
N_{x}=\frac{\partial^{2} \Phi}{\partial y^{2}} ; N_{y}=\frac{\partial^{2} \Phi}{\partial x^{2}} ; N_{x y}=\frac{\partial^{2} \Phi}{\partial x \partial y} .
$$

Alongside with components $\varepsilon_{x}, \varepsilon_{y}$ and $\varepsilon_{\mathrm{xy}}$ (3) we shall enter also mean is thickness $h$ components $\overline{\varepsilon_{x}}, \overline{\varepsilon_{y}}$ and $\overline{\varepsilon_{x y}}$. They can be considered as components of deformation of a median surface [7]:

$$
\left.\begin{array}{l}
\overline{\varepsilon_{x}}=\frac{1}{h} \int_{-h / 2}^{h / 2} \varepsilon_{x} d z=\frac{1}{E h}\left(1+\Pi^{*}\right)\left(N_{x}-\mu N_{y}\right) \\
\overline{\varepsilon_{y}}=\frac{1}{h} \int_{-h / 2}^{h / 2} \varepsilon_{y} d z=\frac{1}{E h}\left(1+\Pi^{*}\right)\left(N_{y}-\mu N_{x}\right) \\
\overline{\varepsilon_{x y}}=\frac{1}{h} \int_{-h / 2}^{h / 2} \varepsilon_{x y} d z=\frac{2(1+\mu)}{E h}\left(1+\Pi^{*}\right) N_{x y}
\end{array}\right\}
$$

Where

$$
N_{x}=\int_{-h / 2}^{h / 2} \sigma_{x} d z, N_{y}=\int_{-h / 2}^{h / 2} \sigma_{y} d z, N_{x y}=\int_{-h / 2}^{h / 2} \sigma_{x y} d z .
$$

The strain components $\overline{\varepsilon_{x}}, \overline{\varepsilon_{\mathrm{y}}}$ and $\overline{\varepsilon_{\mathrm{xy}}}$ are given by the nonlinear relations [7]:

$$
\left.\begin{array}{l}
\bar{\varepsilon} x_{x}=\frac{\partial u}{\partial x}-k_{x} W+\frac{1}{2}\left[\frac{\partial W}{\partial x}\right]^{2}, \\
\overline{\varepsilon_{y}}=\frac{\partial v}{\partial y}-k_{y} W+\frac{1}{2}\left[\frac{\partial W}{\partial y}\right]^{2}, \\
\overline{\varepsilon_{x y}}=\frac{\partial u}{\partial y}+\frac{\partial v}{\partial x}+\frac{\partial W}{\partial x} \frac{\partial W}{\partial y},
\end{array}\right\}
$$

where $u(x, y), v(x, y) \quad$-tangential displacement of points of a median surface, $\kappa_{x}, \kappa_{y}$ - main curvatures of a unreformed median surface.

In Eq.(1), q is the aerodynamic pressure loading due to the uniform supersonic flow. For high Mach numbers this pressure is given approximately by the usual quasi-steady expression [14] 
$-q=\mathrm{B} \frac{\partial W}{\partial t}+B V \frac{\partial W}{\partial x}+B_{1} V^{2}\left(\frac{\partial W}{\partial x}\right)^{2}$.

(4)

Where

$$
\mathrm{B}=æ \frac{p_{\infty}}{V_{\infty}} ; \mathrm{B}_{1}=æ(æ+1) \frac{P_{\infty}}{4 V_{\infty}^{2}} ;
$$

$p_{\infty}$-pressure; $V_{\infty}$ is the speed of sound.

\section{SOLUTION OF THE STRESS FUNCTION}

The stress function $\Phi$ and $W$ - transverse displacement of shells is expanded in a series of functions

$$
\begin{aligned}
\Phi(x, y, t) & =\sum_{n}^{\infty} \sum_{m}^{\infty} \Phi_{n m}(t) \sin \frac{n \pi x}{L} \cos \frac{m y}{R} \\
W(x, y, t) & =\sum_{n}^{\infty} \sum_{m}^{\infty} W_{n m}(t) \sin \frac{n \pi x}{L} \cos \frac{m y}{R}
\end{aligned}
$$

So that only the modes Bubnov-Galerkin method is used to transform Eq.(2) into a set of simultaneous IDE for $\Phi_{\mathrm{k} 1}$ :

$$
\begin{aligned}
& A_{k l} \Phi_{k l}(t)=E\left(1-R^{*}\right) \times \\
& \times\left\{\sum_{n, i}^{\infty} \sum_{m, r}^{\infty} C_{k \ln m i r} W_{n m} W_{i r}+R D_{k l} W_{k l}\right\}
\end{aligned}
$$

The array $A_{k l}$ possesses a unique in verse $A_{k l}{ }^{-1}$ that can be found numerically. The solution for $\Phi_{\mathrm{kl}}$ then has the form

$$
\begin{aligned}
& \Phi_{k l}(t)=E\left(1-R^{*}\right) A_{k l}^{-1} \times \\
& \times\left\{\sum_{n, i}^{\infty} \sum_{m, r}^{\infty} C_{k \ln \operatorname{mir}} W_{n m} W_{i r}+R D_{k l} W_{k l}\right\}
\end{aligned}
$$

\section{MODAL DYNAMIC EQUATIONS}

Substituting the stress function solution (5) are substituted along with the modal expansion (6) for $W$ into the shells equation of motion (1) and applying Bubnov-Galerkin method gives the ordinary nonlinear IDE :

$$
\begin{aligned}
& \frac{D}{h}\left(1-R^{*}\right)\left[\left(\frac{k \pi}{L}\right)^{2}+\left(\frac{l}{R}\right)^{2}\right]^{2} W_{k l}= \\
& -\frac{1}{R}\left(\frac{k \pi}{L}\right)^{2} \Phi_{k l}+\frac{E \pi}{L^{2} R^{2}} \times \\
& \times \sum_{n, i, j}^{\infty} \sum_{m, r, s}^{\infty} a_{k \ln m i r j s} W_{n m}\left(1-R^{*}\right) W_{i r} W_{j s}+
\end{aligned}
$$

$$
\begin{aligned}
& +\frac{E \pi}{L^{2} R} \sum \sum W_{n m} F_{k \ln m i r}\left(1-R^{*}\right) \times \\
& \times W_{i r}-\rho \ddot{W}_{k l}-\frac{B}{h} \dot{W}_{k l}-\frac{2 B V}{L h} \times \\
& \sum_{n=1}^{\infty} n\left(\gamma_{k-n}+\gamma_{k+n}\right) W_{n l}- \\
& -\frac{B_{1} V^{2}}{h} \cdot \frac{\pi}{2 L^{2}} \sum_{n, i}^{\infty} \sum_{m, r}^{\infty} \Gamma_{k \ln m i r} W_{n m} W_{i r}
\end{aligned}
$$

Introducing these parameters,

$$
\frac{W}{h} ; \frac{\mathrm{V}_{\infty} t}{L} \text {, and } \frac{L}{\mathrm{~V}_{\infty} t} R(t),
$$

and keeping the previous designations, the equations (8) takes the non-dimensional forms as follows:

$$
\begin{aligned}
& \ddot{W}_{k l}+\lambda^{4} \Omega^{2}\left\{\left[\left(\frac{k}{\lambda}\right)^{2}+\left(\frac{l}{\pi}\right)^{2}\right]^{2}+\right. \\
& \left.+\frac{12\left(1-\mu^{2}\right)}{\pi^{2} \beta_{1}^{2}}\left(\frac{k}{\lambda}\right)^{2} E_{k l}\right\}\left(1-R^{*}\right) W_{k l}+ \\
& +\beta_{1} \pi^{2} M_{E}^{2} \sum_{n, i}^{\infty} \sum_{m, r}^{\infty} K_{k \ln m i r}\left(1-R^{*}\right) W_{n m} W_{i r}- \\
& -\frac{12 \lambda^{2}\left(1-\mu^{2}\right) \Omega^{2}}{\pi^{3}} \sum_{n, i, j}^{\infty} \sum_{m, r, s}^{\infty} a_{k \ln m i r j s} W_{n m} \times \\
& \left(1-R^{*}\right) W_{i r} W_{j s}-\pi \beta_{1} M_{E}^{2} \sum_{n, i}^{\infty} \sum_{m, r}^{\infty} F_{k \ln m i r} W_{n m} \times \\
& \times\left(1-R^{*}\right) W_{i r}+M \dot{W}_{k l}+2 M M^{*} \sum_{n=1}^{\infty} \gamma_{k n} W_{n l}+ \\
& +\frac{M_{1} M^{* 2}}{2} \pi \sum_{n, i}^{\infty} \sum_{m, r}^{\infty} \Gamma_{k \ln m i r} W_{n m} W_{i r}=0,
\end{aligned}
$$

where

$$
\begin{aligned}
& \gamma_{k n}=n\left(\gamma_{n+k}-\gamma_{n-k}\right) ; \\
& \gamma_{k}= \begin{cases}\frac{\alpha_{k}}{k}, & \mathrm{k}-\text { odd } \\
0, & \mathrm{k}=0 \text { or } \mathrm{k} \text { - even, }\end{cases} \\
& \Omega^{2}=\frac{\pi^{4}}{12\left(1-\mu^{2}\right)}{ }_{E}^{2}\left(\frac{h}{L}\right)^{2} \text {, } \\
& M=ж M_{p}^{2}\left(\frac{L}{h}\right), M_{1}=ж(ж+1) \frac{M_{p}^{2}}{4} ; \quad \lambda=\frac{L}{R} ; \\
& M^{*}=\frac{V}{V_{\infty}} \text { - Mach number; } M_{E}=\sqrt{\frac{E}{\rho V_{\infty}^{2}}} \text {; } \\
& M_{p}=\sqrt{\frac{P_{\infty}}{\rho V_{\infty}^{2}}}, \quad \beta_{1}=\frac{h}{R} ; \quad \gamma_{k n}, \quad \Gamma_{k l n m i r},
\end{aligned}
$$


$a_{\text {klnmir }}, \quad K_{k l n m i r}, F_{\text {klnmir }}-\quad$ non-dimensional coefficients [11].

Equations (9) allow conducting qualitative analysis of solving the problems of the flutters of the viscous-elastic cylindrical shells.

\section{NUMERICAL RESULTS}

Integration of the system (9) of KoltunovRjanitsin kernel $\left(R(t)=A \exp (-\beta t) t^{\alpha-1}, 0<\alpha<1\right)$ was made by numerical method [12]. According to this method the equation (9) twice integrating over $t$ from 0 to $t$, then the formula of numerical integration will as follows:

For this purpose we shall write it in the integral form, then the formula of numerical integration will look like as follows:

$$
\begin{aligned}
& W_{i k l}=\frac{1}{1+A_{i} M}\left\{W_{o k l}+\left(\dot{W}_{o k l}+M W_{o k l}\right) \cdot t_{i}-\right. \\
& -\sum_{j=0}^{i-1} A_{j}\left[M W_{j k l}-\left(t_{i}-t_{j}\right)\left[-2 M M^{*} \sum_{n=1}^{N} \gamma_{k n} W_{j n l}-\right.\right. \\
& -\lambda^{4} \Omega^{2}\left(\left(\left(\frac{k}{\lambda}\right)^{2}+\left(\frac{l}{\pi}\right)^{2}\right)^{2}+\frac{12\left(1-\mu^{2}\right)}{\pi^{2} \beta_{1}^{2}}\left(\frac{k}{\lambda}\right)^{2} E_{k l}\right) \times \\
& {\left[W_{j k l}-\frac{A}{\alpha} \sum_{s=0}^{j} B_{s} e^{-\beta t_{s}} W_{j-s k l}\right]+\frac{12 \lambda^{2}\left(1-\mu^{2}\right) \Omega^{2}}{\pi^{3}} \times} \\
& \times \sum_{\substack{n, i_{1}, j_{1}=1}}^{N} \sum_{\substack{m, r_{1} \\
s_{1}=1}}^{P} a_{k \ln m i \eta r_{j} s_{1}} W_{j n m}\left[W_{j i_{1} r} W_{j j s_{1} s_{1}}\right. \\
& \left.-\frac{A}{\alpha} \sum_{s=0}^{j} B_{s} e^{-\beta t_{s}} W_{j-s i_{1} r} W_{j-s j_{j} s_{1}}\right]+ \\
& +\beta_{1} \pi M_{E}^{2} \sum_{n, i_{1}=1}^{N} \sum_{m, r=1}^{P}\left(F_{k \ln m i r}-\pi K_{k \ln m i_{1} r}\right) W_{j n m} \times \\
& \times\left[W_{j i_{1} r}-\frac{A}{\alpha} \sum_{s=0}^{j} B_{s} \times e^{-\beta t_{s}} W_{j-s i_{1} r}\right]- \\
& \left.\left.\left.-\frac{M_{1} M^{*^{2}}}{2} \pi \sum_{n, i_{1}=1}^{N} \sum_{m, r=1}^{P} \Gamma_{k \ln m i_{1} r} W_{n m} W_{i_{1} r}\right]\right]\right\} \text {, }
\end{aligned}
$$

$$
i=1,2, \ldots ; \quad n=\overline{1, \mathrm{~N}}, \quad m=\frac{(10)}{1, \mathrm{P}}
$$

where $A_{j}, B_{s}$ - numerical coefficient which are not dependent on selection of integrands and receiving different values depending on the used quadrature formulas.

On the basis of algorithm (10), is created of the programs in algorithmic language "Turbo-Pascal".

The results of calculations, conducted by the formulae (10) for shells, flowed by supersonic gas with parameters $p_{\infty}=1,014 \mathrm{~kg} / \mathrm{sm}^{2}, \quad æ=1,4$ and $V_{\infty}=340 \mathrm{~m} / \mathrm{s}$ are given in Table .

As a criterion, determining the critical speed $V_{c r}$ we take condition that at the speeds amplitude of oscillations is changed by harmonically law. At more supercritical speeds oscillatory motions take place in intensive amplitude that can lead to damage of the construction. At $V<V_{c r}$ the oscillatory amplitude is attenuated [15].

The analysis of the results given in the table shows that the coefficient $V_{c r}$ is elastic $(A=0)$ and viscoelastic $(A=0,1)$ according to $780 \mathrm{~m} / \mathrm{s}$ and 423 $\mathrm{m} / \mathrm{s}$. Viscoelastic properties of the material lead to reduction of the flutter speed.

Table. Dependences of critical speeds of visco-elastic shells from physico- mechanical and geometrical parameters

\begin{tabular}{|l|l|l|l|l|l|l|}
\hline A & $\alpha$ & $\beta$ & $\beta_{1}$ & $L / h$ & $m$ & $V_{c r}(\mathrm{~m} / \mathrm{s})$ \\
\hline 0.0 & & & & & & 780 \\
0.001 & & & & & & 687 \\
0.01 & 0.25 & 0.05 & $1 / 500$ & 4000 & 6 & 555 \\
0.1 & & & & & & 423 \\
\hline & 0.1 & & & & & 505 \\
0.01 & 0.5 & 0.05 & $1 / 500$ & 4000 & 6 & 550 \\
& 0.75 & & & & & 575 \\
\hline 0.01 & 0.25 & 0.1 & $1 / 500$ & 4000 & 6 & 540 \\
& & 0.01 & & & & 545 \\
\hline & & & & & & 380 \\
0.01 & 0.25 & 0.05 & $1 / 250$ & 4000 & 6 & 875 \\
& & & & & & 1230 \\
\hline & & & $1 / 250$ & & & 635 \\
0.1 & 0.25 & 0.05 & $1 / 400$ & 2500 & 6 & 393 \\
& & & $1 / 450$ & & & 350 \\
\hline \multirow{2}{*}{0.01} & \multirow{2}{*}{0.25} & 0.05 & $1 / 250$ & 2200 & 6 & 815 \\
& & & & 2500 & & 547 \\
\hline & & & & & 2 & 1053 \\
& & & & & 4 & 1010 \\
0.01 & 0.25 & 0.05 & $1 / 500$ & 4000 & 6 & 555 \\
& & & & & 8 & 605 \\
& & & & & 10 & 1000 \\
\hline
\end{tabular}

Increasing the $\alpha$ parameter leads to changing $V_{c r}$. The research was conducted at $\alpha=0,1 ; 0,5 ; 0,75$. It's clear that increasing the parameter $\alpha$ from 0,1 to 0,75 is accompanied by increasing the critical number $V_{c r}$ of the flutter from $V_{c r}=505 \mathrm{~m} / \mathrm{s}$ to $V_{c r}=575 \mathrm{~m} / \mathrm{s}$.

It was observed the influence of the parameter $L / h$ to shells. Increasing the parameter $L / h$ from 2000 to 2500 leads to reducing $V_{c r}$ up to 49,4 percent. 
The finding out of a role of number of surges $m$ in a circumferential direction introduces large concern for problems of a flutter of shells. At first, knowing a role of number $m$ it is possible to eliminate known uncertainty of the problem of a flutter, which is caused by availability of $m$ in input equations. Secondly, having found out the role of $m$, it is possible to penetrate more deeply into an entity of the flutter phenomenon of shells. However, there is no general analytical and authentic expression for calculus of number $m$ in problems of flutter of shells. Usually each concrete problem at first solved for a number of values $m$ and select that value $m_{\min }$, which is corresponds to minimum critical speed of a flutter (see tab. ). All further calculi are carried out, using the obtained value of $m_{m i n}$.

From the presented analysis it is visible, that to minimum critical speed of a flutter for a circumferential cylindrical shell there corresponds number of surges in a circumferential direction, equal to six. This number of surges $m_{\min }$ also depends on the sizes and characteristics of shell material.

\section{CONCLUSION}

Calculative experiments showed, that the in influence of attenuation parameter $\beta$ of kernel to critical speed of the shell flutter in comparison with the viscosity $A$ parameter and singularity $\alpha$ were negligible that confirms the known conclusion-exponential kernel of relaxation can't describe the material construction properties fully. The negligible reducing the singularity parameter leads to substantial reducing the critical speed of the flutter. Consequently taking in consideration of this effect at the aircraft construction projecting has great importance, as the less the singularity parameter of the material, the higher intensity of dissipative processes in these constructions.

\section{REFERENCES}

[1] Kabulov V.K. Algorithmization in mechanics of continua. Tashkent. 1979. 304 p. (in Russian).

[2] Kabulov V.K. Algorithmization theory of elasticity and deformation theory of strength. Tashkent. 1966. 391 p. (in Russian).

[3] Kabulov V.K ., Babamurodov K.Sh.

Calculation of sandwich shells by computer.

Tashkent. 1970. 334 p. (in Russian).

[4] Dowell, E.H., "Panel Flutter: A Review of the Aeroelastic stability of Plates and Shells," AIAA Journal, Vol.8, No. 2, 1970, pp. 385-399.

[5] Bismarck-Nasr, M.H., "Finite Element Method Applied to the Supersonic Flutter of Circular Cylindrical Shells, " International Journal for
Numerical Methods in Engineering. Vol. 10, No.2, 1976, pp. 423-435.

[6] Mei, C., "A Finite Element Approach for Nonlinear Panel Flutter," AIAA Journal, Vol. 15, No. 8, 1977, pp. 1107-1110.

[7] Bolotin, V.V., Nonconservative Problems of the Theory of Elastic Stability, Moscow. 1961 (in Russian).

[8] Volmir, A.S., Stability of Defortable Systems, Nauka, Moscow,1967 (in Russian).

[9] Bogdanovich, A.E. Nonlinear Problems of dynamic of cylindrical composite shells. Riga: Zinathe.-1987 (in Russian).

[10] Tamuzs, V.P. and Teters, G.A.," Problem of mechanics of Composite Materials, "Mechanics of Composite Materials J. No. 1, 1979, pp. 34-45 (in Russian).

[11] Khudayarov, B.A., Nonlinear flutter of the viscoelastic plates and cylindrical panels. Avtoreferat of diss. On candidate physicsmathematics science. Tashkent. Uzbekistan. 1998. (in Russian).

[12] Badalov, F. B., Eshmatov, H., Yusupov M., “ One method of solution of system of integro differential the problems of viscoelasticity," Journal of Applied Mathematics and Mechanics. Moscow. Vol. 51, No. 5. 1987, pp. 867-871. (in Russian).

[13] Badalov, F. B., Methods solution of integra and integra-differential eduqation inherited of the Theory of viscoelasticity. Tashkent. 1987. (in Russian).

[14] Ilyushin A.A., "The law of flat sections in aerodynamics of fair supersonic speeds," Journal of Applied Mathematics and Mechanics. Moscow. Vol. 20, No. 6. 1956, pp. 733-755. (in Russian).

[15] Verlan A.F., Eshmatov Kh., Khudayarov B.A., Bobonazarov Sh.P. "Numerical solution of nonlinear problem of viscoelastic system dynamics". Electronic simulation J. Vol. 26. No3, 2004, pp.3-14.

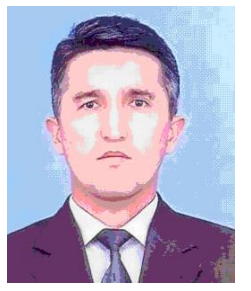

Khudayarov Bakhtiyar Alimovich

Associate of Professor of chair "Higher mathematics" of Tashkent institute of irrigation and melioration. Academic degree and status: Candidate of physicmathematics science, assistantprofessor. Year of birth: 1966.

Address: Chilanzar -16 -24-56, Tashkent, 700135, Uzbekistan.

Phone: (998712) 76-04-02 (home), (99871) 137-0986 (business).

E-mail: bakht-flpo@yandex.ru

Field of research: panel flutter and classic flutter. 